\title{
A survey of dynamical percolation
}

\author{
Jeffrey E. Steif \\ Dedicated to the memory of Oded Schramm, who has been a great inspiration to me and with \\ whom it has been a great honor and privilege to work.
}

\begin{abstract}
Percolation is one of the simplest and nicest models in probability theory/statistical mechanics which exhibits critical phenomena. Dynamical percolation is a model where a simple time dynamics is added to the (ordinary) percolation model. This dynamical model exhibits very interesting behavior. Our goal in this survey is to give an overview of the work in dynamical percolation that has been done (and some of which is in the process of being written up).
\end{abstract}

Mathematics Subject Classification (2000). 60K35.

Keywords. Percolation, exceptional times.

\section{Contents}

1. Introduction

1.1. The ordinary percolation model

1.2. The dynamical percolation model

1.3. Outline of paper

2. Dynamical percolation: first results

2.1. Away from criticality.

2.2. Graphs with exceptional times exist.

2.3. High dimensional Euclidean case

3. Exceptional times of percolation: the tree case

4. Noise sensitivity, noise stability, influence and the Fourier spectrum

4.1. Definition of noise sensitivity, noise stability and some examples

4.2. The noise sensitivity exponent, the noise stability exponent, and influence.

Research partially supported by the Swedish Natural Science Research Council and the Göran Gustafsson Foundation for Research in Natural Sciences and Medicine. 
5. Critical exponents for percolation 15

6. Exceptional times and positivity of the noise sensitivity exponent for the hexagonal lattice

7. The exact Hausdorff dimension of exceptional times and the exact noise sensitivity exponent for the hexagonal lattice 19

8. Sensitivity of the infinite cluster in critical percolation 21

9. Dynamical percolation and the incipient infinite cluster 22

9.1. The incipient infinite cluster 22

9.2. The incipient infinite cluster and dynamical percolation 23

10. The scaling limit of planar dynamical percolation 24

11. Dynamical percolation for interacting particle systems 27

References $\quad 28$

Acknowledgments

\section{Introduction}

Within the very large and beautiful world of probability theory, percolation theory has been an especially attractive subject, an area in which the major problems are easily stated but whose solutions, when they exist, often require ingenious methods. Dynamical percolation is a model where we add a simple time dynamics to the percolation model. It happened to have turned out that this new model is very rich and much can be said about it. It is hoped that this survey will provide a good guide to the literature from which point the reader can study the various papers on the subject of dynamical percolation.

\subsection{The ordinary percolation model}

In the standard percolation model, we take an infinite connected locally finite graph $G$, fix $p \in[0,1]$ and let each edge (bond) of $G$ be, independently of all others, open with probability $p$ and closed with probability $1-p$. Write $\pi_{p}$ for this product measure. In percolation theory, one studies the structure of the connected components (clusters) of the random subgraph of $G$ consisting of all sites and all open edges. The first question that can be asked is the existence of an infinite connected component (in which case we say percolation occurs). Writing $\mathcal{C}$ for this latter event, Kolmogorov's 0-1 law tells us that the probability of $\mathcal{C}$ is, for fixed $G$ and $p$, either 0 or 1 . Since $\pi_{p}(\mathcal{C})$ is nondecreasing in $p$, there exists a critical probability $p_{c}=p_{c}(G) \in[0,1]$ such that

$$
\pi_{p}(\mathcal{C})= \begin{cases}0 & \text { for } p<p_{c} \\ 1 & \text { for } p>p_{c} .\end{cases}
$$

At $p=p_{c}$, we can have either $\pi_{p}(\mathcal{C})=0$ or $\pi_{p}(\mathcal{C})=1$, depending on $G$. Site percolation is defined analogously where the vertices are retained independently with probability $p$ and all the edges between retained vertices are retained. 
For the $d$-dimensional cubic lattice, which is the graph classically studied, the standard reference is [16. For the study of percolation on general graphs, see [41. For a study of critical percolation on the hexagonal lattice, see [54].

While a tremendous amount is known about this model, we highlight some key facts. In the following, $\mathbb{Z}^{d}$ denotes the $d$-dimensional integers with the usual graph structure where points at distance 1 are neighbors.

- For $\mathbb{Z}^{2}$, Harris 22] established in 1960 that there is no percolation at $1 / 2$ and Kesten 28, established in 1980 that there is percolation for $p>1 / 2$. In particular, we see there is no percolation at the critical value for $\mathbb{Z}^{2}$.

- For $\mathbb{Z}^{d}$ with $d \geq 19$, Hara and Slade 21 established that there is no percolation at the critical value. (Percolation connoisseurs will object to this description and say that they, in addition, essentially established this result for $d \geq 7$.)

- Aizenman, Kesten and Newman 2] proved that on $\mathbb{Z}^{d}$, when there is percolation, there is then a unique infinite cluster. Later Burton and Keane 8 ] established this in much greater generality but more importantly found a much simpler proof of this theorem.

We end this subsection with an important open question.

- For $\mathbb{Z}^{d}$, for intermediate dimensions, such as $d=3$, is there percolation at the critical value?

\subsection{The dynamical percolation model}

Häggström, Peres and Steif [19 initiated the study of dynamical percolation. In fact, Olle Häggström and I came up with the model inspired by a question that Paul Malliavin asked at a lecture I gave at the Mittag Leffler Institute in 1995. This model was invented independently by Itai Benjamini. In this model, with $p$ fixed, the edges of $G$ switch back and forth according to independent 2-state continuous time Markov chains where closed switches to open at rate $p$ and open switches to closed at rate $1-p$. Clearly, $\pi_{p}$ is the unique stationary distribution for this Markov process. The general question studied in dynamical percolation is whether, when we start with distribution $\pi_{p}$, there exist atypical times at which the percolation structure looks markedly different than that at a fixed time. In almost all cases, the term "markedly different" refers to the existence or nonexistence of an infinite connected component. Dynamical percolation on site percolation models is defined analogously.

It turns out that dynamical percolation is a very interesting model which exhibits quite new phenomena. It also connects up very much with the recent and important developments in 2-d critical percolation.

In the paper [17, Häggström gives a description of the early results in dynamical percolation and some open questions; most of these earlier results will be repeated here. We also mention the following upcoming paper 13 by Garban which will discuss Oded Schramm's contributions to the area of noise sensitivity; this paper will have some overlap with the present paper. 


\subsection{Outline of paper}

In Section 2, we will start with a discussion of some of the first results obtained for dynamical percolation including (1) "triviality away from criticality", (2) the existence of graphs with "exceptional times" and (3) nonexistence of "exceptional times" for high dimensional lattices. In Section 3, we present the fairly refined results that have been obtained for trees that do not percolate at criticality in ordinary percolation.

We present in Section 4 the basic elements of noise sensitivity and influence together with the Fourier spectrum which is the key tool for the analysis of these concepts. These concepts are both interesting in themselves and are key in the "second moment method" approach to proving the existence of exceptional times for dynamical percolation in 2 dimensions as well as to computing the Hausdorff dimension of the set of exceptional times. Section 5 briefly reviews results concerning critical exponents for ordinary critical percolation since these are crucial to the study of dynamical percolation in 2 dimensions.

In Section 6, we outline the proofs from [51] for the hexagonal lattice of the existence of exceptional times for dynamical percolation (which also yields a nonsharp lower bound on the Hausdorff dimension) and obtain a lower bound for the noise sensitivity exponent for crossing probabilities. (For the square lattice, positivity of the noise sensitivity exponent was established but not the existence of exceptional times.) This method managed to estimate the Fourier spectrum well enough to obtain the above two results. However, the estimates of the spectrum that this method led to were not sharp enough to yield the exact noise sensitivity exponent or the exact dimension of the set of exceptional times. We present the results from 14 in Section 7 These yield for the hexagonal lattice the exact dimension of the set of exceptional times for dynamical percolation and the exact noise sensitivity exponent for crossing probabilities. (For the square lattice, existence of exceptional times for dynamical percolation as well as sharp noise results in terms of the number of pivotals is also established.) These arguments are based on a completely different method to analyze the Fourier spectrum which turns out to be sharp.

Essentially all the results above dealt with graphs which do not percolate at criticality. When dealing with graphs which do percolate at criticality and asking if there are exceptional times of non-percolation, the structure of the problem is very different. A number of results in this case are presented in Section 8 .

In Section 9, a relationship between the incipient infinite cluster (in ordinary percolation) and the structure of the infinite cluster at exceptional times in dynamical percolation is presented. Recent work on the scaling limit of dynamical percolation on the hexagonal lattice is discussed in Section 10, Finally, in Section 11. dynamical percolation for certain interacting particle systems are discussed.

We end the introduction by mentioning that there are a number of other papers, such as [3, 24] and 23], dealing with the existence of certain "exceptional times" for various models. On an abstract level, the questions we are asking concerning 
exceptional behavior are equivalent to questions concerning the polarity or nonpolarity of certain sets for our (Markovian) dynamical percolation model; see [12. Sometimes, the exceptional objects one looks for are of a more general nature such as in [5] and [1].

\section{Dynamical percolation: first results}

This section will discuss a selected subset of the results that were obtained in 19 and [49, which were the first two papers on dynamical percolation.

\subsection{Away from criticality.}

The first very elementary result is the following and contained in 19]. Let $\mathcal{C}_{t}$ be the event that there is an infinite cluster at time $t$ and $\mathrm{P}_{p}$ denote the probability measure governing the whole process when parameter $p$ is used and the graph is understood.

Proposition 2.1. 19] For any graph $G$ we have

$$
\begin{array}{clll}
\mathrm{P}_{p}\left(\left(\neg \mathcal{C}_{t}\right) \text { occurs for every } t\right)=1 & \text { if } & p<p_{c}(G) \\
\mathrm{P}_{p}\left(\mathcal{C}_{t} \text { occurs for every } t\right)=1 & \text { if } & p>p_{c}(G) .
\end{array}
$$

Outline of proof: For the first part, we choose $\delta$ sufficiently small so that the set of edges which are open at some time during a time interval of length $\delta$ is still subcritical. Then on each " $\delta$-interval" there are no exceptional times of percolation and we use countable additivity. The second part is proved in the same way.

This result suggests that the only interesting parameter is the critical one and this is essentially true. However, before restricting ourselves in the continuation to the critical case, we do mention two results for the supercritical case, both from [49].

The first result is a version for dynamical percolation of the uniqueness of the infinite cluster.

Theorem 2.2. 49] Consider dynamical percolation with parameter $p>p_{c}$ on the $d$-dimensional cubic lattice $\mathbb{Z}^{d}$. Then a.s., for all times $t$, there exists a unique infinite cluster.

Proposition 2.3. 49] Let $\Gamma$ be any infinite tree. If $p \in\left(p_{c}(\Gamma), 1\right)$, then a.s., there exist infinitely many infinite clusters for all $t$.

\subsection{Graphs with exceptional times exist.}

We now stick to the critical case. The following result says that dynamical percolation can exhibit exceptional times which then insures that this model is interesting.

Theorem 2.4. 19. There exists a $G_{1}$ which does not percolate at criticality but such that there exist exceptional times of percolation.

There also exists a graph $G_{2}$ which percolates at criticality but such that there exist exceptional times of nonpercolation. 
These graphs were obtained by replacing the edges of $\mathbb{Z}^{2}$ by larger and larger finite graphs which simulated having the original edge move back and forth quickly. These graphs have unbounded degree. Many examples with bounded degree were later discovered and will be discussed in later sections.

\subsection{High dimensional Euclidean case}

Once we have the existence of such examples, it is natural to ask what happens on the standard graphs that we are familiar with. The following result answers this for the cubic lattice in high dimensions. (Recall Hara and Slade 21] proved that ordinary percolation does not percolate at criticality in this case.)

Theorem 2.5. [19] For the integer lattice $\mathbb{Z}^{d}$ with $d \geq 19$, dynamical critical percolation has no exceptional times of percolation.

The key reason for this is a highly nontrivial result due to Hara and Slade, that says that if $\theta(p)$ is the probability that the origin percolates when the parameter is $p$, then

$$
\theta(p)=O\left(p-p_{c}\right) .
$$

In fact, Theorem 2.5 was shown to hold under the assumption (2.1).

Outline of proof: We use a first moment argument together with the proof method of Proposition 2.1. We break the time interval $[0,1]$ into $n$ intervals each of length $1 / n$. If we fix one of these intervals, the set of edges which are open at some time during this interval has density about $p_{c}+1 / n$. Hence the probability that the origin percolates with respect to these set of edges is by (2.1) at most $O(1 / n)$. It follows that the expected number of these intervals where this occurs is at most $O(1)$. It can then be argued using Fatou's Lemma that a.s. there are at most finitely many exceptional times during $[0,1]$ at which the origin percolates. To go from there to no exceptional times can either be done by using some rather abstract Markov process theory or, as was done in the paper, by hand, which was not completely trivial.

It is known, due to Kesten and Zhang 32, that (2.1) fails for $\mathbb{Z}^{2}$. The question of whether there are exceptional times for critical dynamical percolation on $\mathbb{Z}^{2}$ was left open in 19]. (Recall there is no percolation at a fixed time in this case.) This question will be resolved in Sections [6] and 7.

\section{Exceptional times of percolation: the tree case}

Although Theorem 2.4 demonstrated the existence of graphs which have exceptional times where the percolation picture is different from that at a fixed time, in [19], a more detailed analysis was done for trees.

To explain this, we first need to give a little background from ordinary percolation on trees, results which are due to Lyons and contained in [39] and [40. Lyons obtained an explicit condition when a given tree percolates at a given value of $p$. This formula becomes simplest when the (rooted) tree is so-called spherically 
symmetric which means that all vertices at a given level have the same number of children, although this number may depend on the given level.

Theorem 3.1. [40] (Case of spherically symmetric trees)

Let 0 be the root of our tree and $T_{n}$ be the number of vertices at the nth level. Then the following hold.

(i) $p_{c}(T)=\left(\liminf _{n} T_{n}^{1 / n}\right)^{-1}$.

(ii) $\mathrm{P}_{p}(0 \leftrightarrow \infty)>0$ if and only if $\sum_{n} \frac{1}{w_{n}}<\infty$ where

$w_{n}:=\mathrm{E}_{p}[$ number of vertices in nth level connected to 0$]=p^{n} T_{n}$.

Note that (i) essentially follows from (ii). In [19], an explicit condition was obtained for when, given a general tree and a value of $p$, there exists for dynamical percolation a time at which percolation occurs. Again the spherically symmetric case is simplest.

Theorem 3.2. [19] (Case of spherically symmetric trees)

Let $w_{n}$ be as in the previous result. Then there exists a.s. a time at which percolation occurs (equivalently with positive probability there exists a time in $[0,1]$ at which the root percolates) if and only if $\sum_{n} \frac{1}{n w_{n}}<\infty$.

Example: If $T_{n} \asymp 2^{n} n^{1 / 2}$, then the critical value is $1 / 2$, there is no percolation at criticality but there are exceptional times at which percolation occurs (which will have dimension $1 / 2$ by our next result). (The symbol $\asymp$ means that the ratio of the two sides is bounded away from zero and infinity by constants.)

Outline of proof: For the "if" direction, we apply the second moment method to the random variables

$$
Z_{n}:=\sum_{v \in T_{n}} U_{v}
$$

where $U_{v}$ is the Lebesgue amount of time during $[0,1]$ that the root is connected to $v$ and $T_{n}$ now denotes the set of vertices at the $n$th level. (The "second moment method" means that one computes both the first and second moments of a given nonnegative random variable $X$ and applies the Cauchy-Schwarz inequality to obtain $P(X>0) \geq\left(E[X]^{2}\right) / E\left[X^{2}\right]$.) For the "only if" direction, we perform a slightly nontrivial first space-time decomposition argument as follows. If there is some $v$ in $T_{n}$ which percolates to the root at some time $t$ during $[0,1]$, we show that one can pick such a random pair $\left(v^{\prime}, t^{\prime}\right)$ in such a way that $E\left[Z_{n} \mid\left(v^{\prime}, t^{\prime}\right)\right]$ is much larger than $E\left[Z_{n}\right]$. This implies that it is very unlikely that such a pair exists.

In [18, it was shown that some of the more refined results in [40] and Theorem 3.2 above together yield that a spherically symmetric tree has an exceptional time of percolation if and only if $\int_{p_{c}}^{1} 1 / \theta(p)<\infty$. (As we will see, this equivalence also turns out to be true for $\mathbb{Z}^{d}$ with $d=2$ or $d \geq 19$.) It was also shown in 18 that for general trees, the "only if" holds but not the "if". 
In the spherically symmetric case, the Hausdorff dimension was also determined in [19].

Theorem 3.3. 19] Consider a spherically symmetric tree and let $w_{n}$ be as in the two previous results. Then the Hausdorff dimension of the set of times at which percolation occurs is given by

$$
\sup \left\{\alpha \in[0,1]: \sum_{n=1}^{\infty} \frac{n^{\alpha-1}}{w_{n}}<\infty\right\} .
$$

While we do not state the results here, we mention that 49] went a good deal further for spherically symmetric trees. What was obtained in [49] was (see Corollary 5.1 there) a "capacity condition" for which subsets of time have the property that with positive probability they contain a percolating time. This is analogous to the well-known Kakutani criterion (in terms of Newtonian capacity) of which subsets in Euclidean space intersect a Brownian motion path with positive probability.

Once we have such a capacity condition, Peres' intersection equivalence theory (45] and [46]) leads to a criterion for when there are exceptional times at which there are at least $k$ infinite clusters. We can in particular construct trees for which there are times at which we have (say) 6 infinite clusters but no times at which there are 7 infinite clusters. In addition, various Hausdorff dimensions of these different exceptional time sets can be computed.

On a much less formal note, my personal feeling is that the set of exceptional times in these cases, very vaguely speaking, might have a similar structure to the set of so-called "slow" points for Brownian motion. In Section 8, we will see a very different type of set of exceptional times for dynamical percolation and I believe that in this latter case, this set might behave more like the set of so-called "fast" points for Brownian motion. Here are two words explaining this vague connection (for those who know these concepts from Brownian motion). For a time point $s$ to be a slow point, it must be the case that $|B(t+s)-B(s)|$ does not go above a certain (well-specified) level for all values of small $t$ while a time $s$ is an exceptional time of percolation if the origin percolates out to all distances. On the other hand, for a time point $s$ to be a fast point, it must be the case that $|B(t+s)-B(s)|$ goes above a certain (well-specified) level for an infinite number of arbitrarily small $t$ while a time $s$ is an exceptional time of non-percolation say for a tree if we have an infinite number of cut-sets which are off. This type of structure looks like something which is called a limsup fractal; see [35].

We end by mentioning that in 33, Khoshnevisan extended to general trees the result of Peres and Steif determining which time sets contain percolating times. Results concerning the Hausdorff dimension of exceptional times for general trees are also obtained in [33. Some of the techniques use methods from [34]. 


\section{Noise sensitivity, noise stability, influence and the Fourier spectrum}

The study of noise sensitivity and noise stability for Boolean functions was initiated in 4. The concepts discussed in this section come from or are motivated by this source. See [44] for a nice survey of noise sensitivity and its applications in theoretic computer science. See also [26] for related matters.

\subsection{Definition of noise sensitivity, noise stability and some examples}

As indicated in the introduction, the notion of noise sensitivity is both an interesting concept in itself and what is needed to carry out the second moment arguments necessary for the results described in Sections 6 and 7

Let $\omega$ be uniformly chosen from the $n$-dimensional discrete cube $\{0,1\}^{n}$ (which we can think of as $n$ fair coin flips) and let $\omega_{\epsilon}$ be $\omega$ but with each bit independently "rerandomized" with probability $\epsilon$. "Rerandomized" means the bit (independently of everything else) rechooses whether it is 1 or 0 , each with probability $1 / 2$. (Of course $\omega_{\epsilon}$ has the same distribution as $\omega$ ).

Let $f:\{0,1\}^{n} \rightarrow\{ \pm 1\}$ or $\{0,1\}$ be arbitrary. We are interested in the covariance between $f(\omega)$ and $f\left(\omega_{\epsilon}\right)$. In most cases of interest, we will have a sequence $\left\{f_{n}\right\}$ where $f_{n}:\{0,1\}^{m_{n}} \rightarrow\{ \pm 1\}$ or $\{0,1\}$ and we are interested in the asymptotic behavior of the covariance above.

Definition 4.1. The sequence $\left\{f_{n}\right\}$ is noise sensitive if for every $\epsilon>0$,

$$
\lim _{n \rightarrow \infty} \mathrm{E}\left[f_{n}(\omega) f_{n}\left(\omega_{\epsilon}\right)\right]-\mathrm{E}\left[f_{n}(\omega)\right]^{2}=0 .
$$

Usually $f$ is an indicator of an event $A$ and this then says that the two events $\{\omega \in A\}$ and $\left\{\omega_{\epsilon} \in A\right\}$ are close to independent for $\epsilon$ fixed and $n$ large. The following notion captures the opposite situation where the two events above are close to being the same event if $\epsilon$ is small, uniformly in $n$.

Definition 4.2. The sequence $\left\{f_{n}\right\}$ is noise stable if

$$
\lim _{\epsilon \rightarrow 0} \sup _{n} \mathrm{P}\left(f_{n}(\omega) \neq f_{n}\left(\omega_{\epsilon}\right)\right)=0 .
$$

It is easy to check that $\left\{f_{n}\right\}$ is both noise sensitive and noise stable if and only if the sequence of variances $\left\{\operatorname{Var}\left(f_{n}\right)\right\}$ goes to 0 . Here are two easy examples where $m_{n}$ is taken to be $n$.

Example 1: $f_{n}(\omega)=\omega_{1}$ (i.e., the first bit).

Example 2: $f_{n}(\omega)$ is the parity of the number of 1's in $\omega$.

It is easy to check that Example 1 is noise stable while Example 2 is noise sensitive. We will see later why these two examples are the two extreme examples. A more interesting example is the following which, while it is not immediately obvious, turns out to be noise stable as shown in [4].

Example 3 (Majority): Let $m_{n}=2 n+1$. Let $f_{n}(\omega)$ be 1 if there is a majority of 1 's and 0 if there is a majority of 0 's. 
A much more interesting example is the following.

Example 4: Let $f_{n}$ be the indicator function of a left to right crossing of the box $[0, n] \times[0, n]$ for critical percolation either for the ordinary lattice $\mathbb{Z}^{2}$ or for the hexagonal lattice. For the hexagonal lattice, the box has to be slightly modified so that it is a union of hexagons. (Note $m_{n}$ is of order $n^{2}$ in this case.)

Theorem 4.3. [4] The sequence $\left\{f_{n}\right\}$ in Example 4 is noise sensitive.

In fact, it was shown that

$$
\lim _{n \rightarrow \infty} \mathrm{E}\left[f_{n}(\omega) f_{n}\left(\omega_{\epsilon_{n}}\right)\right]-\mathrm{E}\left[f_{n}(\omega)\right]^{2}=0
$$

even when $\epsilon_{n}$ goes to 0 with $n$ provided that $\epsilon_{n} \geq C / \log (n)$ for a sufficiently large $C$. Clearly for any sequence of Boolean functions, if $\epsilon_{n}$ goes to 0 sufficiently fast, we have

$$
\lim _{n \rightarrow \infty} \mathrm{P}\left(f_{n}(\omega) \neq f_{n}\left(\omega_{\epsilon_{n}}\right)\right)=0
$$

for the trivial reason that in that case $\mathrm{P}\left(\omega \neq \omega_{\epsilon_{n}}\right) \rightarrow 0$.

4.2. The noise sensitivity exponent, the noise stability exponent, and influence.

For sequences $\left\{f_{n}\right\}$ which are noise sensitive, it might be hoped that (4.1) is still true when $\epsilon_{n}$ decays as some power of $1 / n$ and this was explicitly asked in [4 for crossings in critical percolation. This suggested "critical exponent" is what we call the noise sensitivity exponent. The following definitions now seem natural.

Definition 4.4. The noise sensitivity exponent $\left(\operatorname{SENS}\left(\left\{f_{n}\right\}\right)\right)$ of a sequence $\left\{f_{n}\right\}$ is defined to be

$$
\sup \left\{\alpha: \text { (4.1) holds with } \epsilon_{n}=(1 / n)^{\alpha}\right\} .
$$

The noise stability exponent $\left(\operatorname{STAB}\left(\left\{f_{n}\right\}\right)\right)$ of a sequence $\left\{f_{n}\right\}$ is defined to be

$$
\inf \left\{\alpha: \text { (4.2) holds with } \epsilon_{n}=(1 / n)^{\alpha}\right\} \text {. }
$$

Remarks:

1. We will see later that $\mathrm{E}\left[f(\omega) f\left(\omega_{\epsilon}\right)\right]-\mathrm{E}[f(\omega)]^{2}$ is nonnegative and decreasing in $\epsilon$. It easily follows that $\mathrm{P}\left(f_{n}(\omega) \neq f_{n}\left(\omega_{\epsilon}\right)\right)$ is increasing in $\epsilon$. From this, it is easy to see that $\operatorname{SENS}\left(\left\{f_{n}\right\}\right) \leq \operatorname{STAB}\left(\left\{f_{n}\right\}\right)$ unless the variances $\operatorname{Var}\left(\left\{f_{n}\right\}\right)$ go to 0 .

2. One might hope that $\operatorname{SENS}\left(\left\{f_{n}\right\}\right)=\operatorname{STAB}\left(\left\{f_{n}\right\}\right)$. First, this can fail for trivial reasons such as the $f_{n}$ 's for even $n$ might have nothing to do with the $f_{n}$ 's for odd $n$. However, this may fail for more interesting reasons. Using $\operatorname{STAB}\left(\left\{A_{n}\right\}\right)$ for $\operatorname{STAB}\left(\left\{I_{A_{n}}\right\}\right)$ and similarly for SENS, if, for example, $A_{n}$ and $B_{n}$ are independent for each $n$, have probabilities near $1 / 2$ and satisfy

$$
\operatorname{SENS}\left(\left\{A_{n}\right\}\right)=\operatorname{STAB}\left(\left\{A_{n}\right\}\right)=a<b=\operatorname{SENS}\left(\left\{B_{n}\right\}\right)=\operatorname{STAB}\left(\left\{B_{n}\right\}\right),
$$

then it is easy to check that

$$
\operatorname{SENS}\left(\left\{A_{n} \cap B_{n}\right\}\right)=a<b=\operatorname{STAB}\left(\left\{A_{n} \cap B_{n}\right\}\right) .
$$

In such a case, for $\epsilon_{n}=(1 / n)^{c}$ with $c \in(a, b)$, the correlation between $\{\omega \in$ $\left.A_{n} \cap B_{n}\right\}$ and $\left\{\omega_{\epsilon_{n}} \in A_{n} \cap B_{n}\right\}$ neither goes to 0 nor to being perfectly correlated. 
The question of under what conditions we have $\operatorname{SENS}\left(\left\{f_{n}\right\}\right)=\operatorname{STAB}\left(\left\{f_{n}\right\}\right)$ turns out to be a fairly subtle one.

3. If $m_{n} \asymp n^{\sigma}$, then it is trivial to check that $\operatorname{STAB}\left(\left\{f_{n}\right\}\right) \leq \sigma$ since if $\epsilon_{n}=$ $(1 / n)^{\sigma+\delta}$ for some fixed $\delta$, then $\mathrm{P}\left(\omega \neq \omega_{\epsilon_{n}}\right) \rightarrow 0$.

4. It is natural to ask for bounds on these exponents for general Boolean functions; this will be discussed at the end of subsection 4.3 .

The next important notion of total influence will give us a more interesting upper bound on the noise stability exponent than that provided by comment 3 above.

Definition 4.5. Given a function $f:\{0,1\}^{n} \rightarrow\{ \pm 1\}$ or $\{0,1\}$, let $I_{i}(f)$, which we call the influence of the $i$ th variable on $f$, be the probability that all the variables other than the $i$ th variable do not determine $f$. I.e., letting $\omega^{i}$ be $\omega$ but flipped in the $i$ th coordinate,

$$
I_{i}(f):=\mathrm{P}\left(\omega: f(\omega) \neq f\left(\omega^{i}\right)\right) .
$$

The total influence, denote by $I(f)$, is defined to be $\sum_{i} I_{i}(f)$. If $f(\omega) \neq f\left(\omega^{i}\right)$, we say that $i$ is pivotal for $f$ and $\omega$ and hence $I(f)$ is the expected number of pivotal bits of $f$.

The following I believe was "known" in the community. The argument below however I first saw given by Christophe Garban in the context of percolation.

Theorem 4.6. Consider a sequence $f_{n}:\{0,1\}^{m_{n}} \rightarrow\{ \pm 1\}$ or $\{0,1\}$ and assume that $I\left(f_{n}\right)=n^{\rho+o(1)}$. Then $\operatorname{STAB}\left(\left\{f_{n}\right\}\right) \leq \rho$.

Proof: We need to show that if $\alpha>\rho$ and $\epsilon_{n}=(1 / n)^{\alpha}$, then (4.2) holds. Let $\omega_{0}, \omega_{1}, \ldots, \omega_{k_{n}}$ be such that one obtains $\omega_{i+1}$ from $\omega_{i}$ by choosing independently a bit at random and rerandomizing it. It is immediate that $\mathrm{P}\left(f_{n}\left(\omega_{i}\right) \neq f_{n}\left(\omega_{i+1}\right)\right)=$ $\frac{I\left(f_{n}\right)}{2 m_{n}}$ from which one gets $\mathrm{P}\left(f_{n}\left(\omega_{0}\right) \neq f_{n}\left(\omega_{k_{n}}\right)\right) \leq \frac{k_{n} n^{\rho+o(1)}}{m_{n}}$. If $\epsilon_{n}=(1 / n)^{\alpha}$, then $\omega_{\epsilon_{n}}$ is sort of like $\omega_{k_{n}}$ with $k_{n}=\frac{m_{n}}{n^{\alpha}}$ and so

$$
\mathrm{P}\left(f_{n}(\omega) \neq f_{n}\left(\omega_{\epsilon_{n}}\right)\right) \sim \mathrm{P}\left(f_{n}\left(\omega_{0}\right) \neq f_{n}\left(\omega_{k_{n}}\right)\right) \leq \frac{m_{n} n^{\rho+o(1)}}{m_{n} n^{\alpha}}
$$

which goes to 0 as $n \rightarrow \infty$ if $\alpha>\rho$. The "sort of like" above and the imprecise $\sim$ are trivial to make rigorous and correct using standard large deviations for binomial random variables.

Remarks:

An example where we have strict inequality is the "majority function" of Example 3. It is easy to check that for this example $\rho$ in Theorem 4.6 is $1 / 2$ but, by the noise stability of this example, we have that $\operatorname{STAB}\left(\left\{f_{n}\right\}\right)=0$. One explanation of the failure of having a converse to Theorem 4.6 in this case is that the expected number of pivotals is not so relevant: the random number $N_{n}$ of pivotals is not at all concentrated around its mean $\mathrm{E}\left[N_{n}\right]=I\left(f_{n}\right) \asymp n^{1 / 2}$ but rather it goes to 0 in probability. 
We will see an alternative proof of Theorem 4.6 using Fourier analysis in the next subsection.

Of the very many nice results in 4, we mention the following one which almost characterizes noise sensitivity in terms of influences. As the authors mention, this result could be used to prove Theorem 4.3 but they instead use a different approach. A function $f$ is montone if $x \leq y$ (meaning $x_{i} \leq y_{i}$ for each $i$ ) implies that $f(x) \leq f(y)$. The proof of the following result uses the Fourier spectrum (see the next subsection) and a notion known as hypercontractivity.

Theorem 4.7. [4] Consider a sequence of Boolean functions $\left\{f_{n}\right\}$. If $\lim _{n \rightarrow \infty} \sum_{i} I_{i}\left(f_{n}\right)^{2}=0$, then the sequence $\left\{f_{n}\right\}$ is noise sensitive. The converse is true if the $f_{n}$ 's are monotone. (Example 2 shows that monotonicity is needed for the converse.)

We end this section by going back to Example 4. It was asked in [4] whether the noise sensitivity exponent for this sequence is $3 / 4$. The heuristic for this guess is the following. An edge (or hexagon) is pivotal if and only if there are 4 disjoint monochromatic paths alternating in color from the edge (or hexagon) to the top, right, bottom and left sides of the box. This is close to the 4-arm exponent which by 53 was proved to be behave like $n^{-5 / 4}$. If boundary terms do not matter much, $I\left(f_{n}\right)$ should then be about $n^{3 / 4}$. One might hope that the number $N_{n}$ of pivotals is quite concentrated around its mean $I\left(f_{n}\right)$; in this direction, it is well-known for example that $\mathrm{E}\left[N_{n}^{2}\right]=O(1) \mathrm{E}\left[N_{n}\right]^{2}$. This gives hope that Theorem 4.6 is now tight in this case and that $3 / 4$ might be both the noise sensitivity and noise stability exponents. In Section 7, we will see that this is indeed the case, but the proof will not go through understanding pivotals but rather through Fourier analysis. This brings us to our next topic.

\subsection{The Fourier spectrum}

It turns out that the best and proper way to analyze the above problems is to use Fourier analysis. The set of all functions $f:\{0,1\}^{n} \rightarrow \mathbb{R}$ is a $2^{n}$ dimensional vector space with orthogonal basis $\left\{\chi_{S}\right\}_{S \subseteq\{1, \ldots, n\}}$ where

$$
\chi_{S}\left(\omega^{1}, \ldots, \omega^{n}\right):= \begin{cases}-1 & \text { if \# of 1's in }\left\{\omega^{i}\right\}_{i \in S} \text { is odd } \\ 1 & \text { if \# of 1's in }\left\{\omega^{i}\right\}_{i \in S} \text { is even. }\end{cases}
$$

So $\chi_{\emptyset} \equiv 1$. We then can write

$$
f:=\sum_{S \subseteq\{1, \ldots, n\}} \hat{f}(S) \chi_{S}
$$

(In fancy terms, the various $\chi_{S}$ 's are the so-called characters on the group $\mathbb{Z}_{2}^{n}$ but everything below will be from first principles). The $\hat{f}(S)$ 's are called the Fourier coefficients.

The reason that $\left\{\chi_{S}\right\}$ is a useful basis is that they are eigenfunctions for the discrete time Markov chain which takes $\omega$ to $\omega_{\epsilon}$. It is an easy exercise to check that

$$
\mathrm{E}\left[\chi_{S}\left(\omega_{\epsilon}\right) \mid \omega\right]=(1-\epsilon)^{|S|} \chi_{S}(\omega)
$$


and that

$$
\mathrm{E}\left[f(\omega) f\left(\omega_{\epsilon}\right)\right]=\mathrm{E}[f(\omega)]^{2}+\sum_{k=1}^{n}(1-\epsilon)^{k} \sum_{|S|=k} \hat{f}(S)^{2} .
$$

This formula which first (I believe) appeared in [4 makes clear the central role played by the Fourier coefficients with respect to questions involving noise. Note that we see the nonnegativity of the covariance between $f(\omega)$ and $f\left(\omega_{\epsilon}\right)$ and that it is decreasing in $\epsilon$ as we had claimed earlier. (I am not aware of any coupling proof of this latter fact; we can of course couple $\omega, \omega_{\epsilon}$ and $\omega_{\epsilon+\delta}$ so that $\omega_{\epsilon}$ agrees with $\omega$ in more places than $\omega_{\epsilon+\delta}$ does but in view of Example 2 in Subsection 4.1, this does not seem to help.) Crucially, we see that the covariance between $f(\omega)$ and $f\left(\omega_{\epsilon}\right)$ is small when most of the "weight" of these coefficients are on the $S$ 's with larger cardinality while the covariance is largest when most of the "weight" of these coefficients are on the smaller $S$ 's.

In Subsection 4.1 Example 1 is the function $\left(1-\chi_{\{1\}}\right) / 2$ while Example 2 is the function $\chi_{\{1, \ldots, n\}}$ from which we now see why these are extreme examples as we mentioned earlier.

We now restrict to $f$ 's which take values in $\{-1,1\}$. The advantage in doing this is that we have (due to the Pythagorean theorem or Parseval's formula)

$$
\sum_{S \subseteq\{1, \ldots, n\}} \hat{f}(S)^{2}=1 .
$$

(For those who do not like the restriction of $\{-1,1\}$ on the range of $f$ since you are interested in indicator functions of events, you can just consider the function which is 1 on the event in question and -1 on its complement and then easily translate back the results below in terms of your events.)

Given the above, we can let $\mathcal{S}$ be a random subset of $\{1, \ldots, n\}$ given by $\mathrm{P}(\mathcal{S}=S)=\hat{f}(S)^{2}$. The idea of looking at this as a probability distribution on the subsets of $\{1, \ldots, n\}$ was proposed in 4 and is called the spectral sample or spectral measure of $f$. (We will not be careful to distinguish between $\mathcal{S}$ and its distribution.) (4.3) can now be written as (with the two expectations being on different spaces)

$$
\mathrm{E}\left[f(\omega) f\left(\omega_{\epsilon}\right)\right]=\mathrm{E}\left[(1-\epsilon)^{|\mathcal{S}|}\right] .
$$

This equation demonstrates the important fact that a sequence $\left\{f_{n}\right\}$ is noise sensitive if and only if the corresponding spectral measures $\left\{\mathcal{S}_{n}\right\}$ satisfy $\left|\mathcal{S}_{n}\right| \rightarrow \infty$ in distribution provided we remove the point mass at $\emptyset$ (which corresponds to subtracting the squared mean).

We now give an alternative proof of Theorem 4.6 which is taken from [14]. 
Proof 14: We need only one thing which we do not prove here which comes from [25]; one can also see a proof of this in [14]. This is that

$$
\mathrm{E}[|\mathcal{S}|]=I(f) \text {. }
$$

It clearly suffices to show that if $\alpha>\rho$ and $\epsilon_{n}=(1 / n)^{\alpha}$, then

$$
\lim _{n \rightarrow \infty} \mathrm{E}\left[f_{n}(\omega) f_{n}\left(\omega_{\epsilon_{n}}\right)\right]=1 .
$$

(4.4), Jensen's inequality and $\mathrm{E}\left[\left|\mathcal{S}_{n}\right|\right]=I\left(f_{n}\right)$ yields

$$
\begin{gathered}
\mathrm{E}\left[f_{n}(\omega) f_{n}\left(\omega_{\epsilon_{n}}\right)\right]=\mathrm{E}\left[\left(1-\epsilon_{n}\right)^{\left|\mathcal{S}_{n}\right|}\right] \geq\left(1-\epsilon_{n}\right)^{\mathrm{E}\left[\left|\mathcal{S}_{n}\right|\right]}=\left(1-\epsilon_{n}\right)^{I\left(f_{n}\right)} \\
=\left(1-(1 / n)^{\alpha}\right)^{n^{\rho+o(1)}} .
\end{gathered}
$$

This goes to 1 since $\alpha>\rho$.

In the context of Theorem 4.6, it would be nice to know under which further conditions we could conclude the reverse inequality and even that $\operatorname{SENS}\left(\left\{f_{n}\right\}\right) \geq \rho$. One sufficient condition is that the distributions of $\left|\mathcal{S}_{n}\right|$, normalized by their means and with the point mass at 0 removed is tight on $(0, \infty)$. (Tightness at $\infty$ follows from Markov's inequality; the key point is tightness near 0 .) We make this precise in the following result. This result is proved by just abstracting an easy small part of an argument from [14.

Theorem 4.8. Assume $f_{n}:\{0,1\}^{m_{n}} \rightarrow\{ \pm 1\}$ and that $I\left(f_{n}\right)=n^{\rho+o(1)}$. Let $\mathcal{S}_{n}$ be the spectral sample corresponding to $f_{n}$. Assume that for every $\gamma>0$, there is $\delta>0$ so that for all $n$

$$
\mathrm{P}\left(\left|\mathcal{S}_{n}\right|<\delta \mathrm{E}\left[\left|\mathcal{S}_{n}\right|\right], \mathcal{S}_{n} \neq \emptyset\right)<\gamma .
$$

Then $\operatorname{SENS}\left(\left\{f_{n}\right\}\right) \geq \rho$.

Proof: We need to show that if $\alpha<\rho$, (4.1) holds when $\epsilon_{n}=(1 / n)^{\alpha}$. The difference in (4.1) is by (4.3) and (4.4) simply $\mathrm{E}\left[\left(1-\epsilon_{n}\right)^{\left|\mathcal{S}_{n}\right|} I_{\mathcal{S}_{n} \neq \emptyset}\right]$. Fix $\gamma>0$ and choose $\delta$ as in the assumption. We have

$$
\mathrm{E}\left[\left(1-\epsilon_{n}\right)^{\left|\mathcal{S}_{n}\right|} I_{\left.\mathcal{S}_{n} \neq \emptyset\right]} \leq \mathrm{P}\left(\left|\mathcal{S}_{n}\right|<\delta \mathrm{E}\left[\left|\mathcal{S}_{n}\right|\right], \mathcal{S}_{n} \neq \emptyset\right)+\left(1-\epsilon_{n}\right)^{\delta \mathrm{E}\left[\left|\mathcal{S}_{n}\right|\right]} .\right.
$$

The first term is at most $\gamma$ for all $n$ by the choice of $\delta$ and the last term goes to 0 since $\delta$ is fixed, $\alpha<\rho$ and using (4.5). Since $\gamma$ was arbitrary, we are done.

It is now interesting to look again at the majority function for which $\rho=$ $1 / 2$ but $\operatorname{SENS}\left(\left\{f_{n}\right\}\right)=\operatorname{STAB}\left(\left\{f_{n}\right\}\right)=0$. In this case, the spectral measures do not satisfy the necessary tightness condition above but rather these distributions normalized by their means approach the point mass at 0 . This follows from the known noise stability of majority, the fact (see Theorem 1.9 in 4]) that stability in general implies tightness at $\infty$ for the unnormalized spectral measures and the fact that the expected value of the spectral size is going to $\infty$. See [4] for details concerning the spectral measure of majority. 
The following is an exercise for the reader which relates the lower tail of the spectral measures with noise sensitivity; Theorem 4.8 already gave some relationship between these.

Exercise 1: Let $f_{n}$ be an arbitrary sequence of Boolean functions with corresponding spectral samples $\mathcal{S}_{n}$.

(i) Show that $\mathrm{P}\left(0<\left|\mathcal{S}_{n}\right| \leq A_{n}\right) \rightarrow 0$ implies that $\mathrm{E}\left[\left(1-\epsilon_{n}\right)^{\left|\mathcal{S}_{n}\right|} I_{\mathcal{S}_{n} \neq \emptyset}\right] \rightarrow 0$ if $\epsilon_{n} A_{n} \rightarrow \infty$.

(ii) Show that $\mathrm{E}\left[\left(1-\epsilon_{n}\right)^{\left|\mathcal{S}_{n}\right|} I_{\left.\mathcal{S}_{n} \neq \emptyset\right]} \rightarrow 0\right.$ implies that $\mathrm{P}\left(0<\left|\mathcal{S}_{n}\right| \leq A_{n}\right) \rightarrow 0$ if $\epsilon_{n} A_{n}=O(1)$.

In particular, $\operatorname{SENS}\left(\left\{f_{n}\right\}\right) \geq \alpha$ if and only if $\mathrm{P}\left(0<\left|\mathcal{S}_{n}\right| \leq n^{\alpha-\delta}\right) \rightarrow 0$ for all $\delta>0$.

Exercise 2: Show that $\operatorname{STAB}\left(\left\{f_{n}\right\}\right) \leq \alpha$ if and only if $\mathrm{P}\left(\left|\mathcal{S}_{n}\right| \geq n^{\alpha+\delta}\right) \rightarrow 0$ for all $\delta>0$.

We end this subsection with a brief discussion of general upper bounds on our noise sensitivity and noise stability exponents. We stick for simplicity and without loss of generality to $m_{n}=n$. We have seen then that 1 is an upper bound for these exponents. On the other hand, the parity function, Example 2, is easily seen to have both these exponents being 1. This question turns out to be much more interesting if we restrict to the important subclass of monotone Boolean functions.

Theorem 4.9. Assume $f_{n}:\{0,1\}^{n} \rightarrow\{ \pm 1\}$ be monotone. Then $\operatorname{STAB}\left(\left\{f_{n}\right\}\right) \leq$ $1 / 2$.

Outline of proof: It is an exercise to check, crucially using the monotonicity, that $I_{i}\left(f_{n}\right)=\left|\hat{f}_{n}(\{i\})\right|$. It follows that $\sum_{i} I_{i}\left(f_{n}\right)^{2} \leq 1$. The Cauchy-Schwartz inequality now yields that $\sum_{i} I_{i}\left(f_{n}\right) \leq \sqrt{n}$. The result now follows from Theorem 4.6.

Remark:

The above theorem can be proved (and has been) without the use of Fourier analysis and is known in various contexts.

Answering a question in 4, it was shown in 42] that the above result is optimal by giving a sequence $\left\{f_{n}\right\}$ of monotone functions with $\operatorname{STAB}\left(\left\{f_{n}\right\}\right)=1 / 2$. By tweaking these examples, one can also obtain a sequence with $\operatorname{SENS}\left(\left\{f_{n}\right\}\right)=1 / 2$.

\section{Critical exponents for percolation}

The exact values for critical exponents for percolation on the hexagonal lattice are a crucial ingredient in the study of exceptional times for dynamical percolation and noise sensitivity for crossing probabilities. We therefore briefly describe these.

Let $A_{R}^{k}$ be the event (for ordinary percolation) that there are $k$ disjoint monochromatic paths from within distance (say) $2 k$ of the origin all of which reach distance $R$ from the origin and such that they are not all of the same color. This is referred to as the $k$-arm event. Let $A_{R}^{k, H}$ be the analogous event but where we restrict to the upper half plane and where the restriction "not all of the same 
color" may be dropped. This is referred to as the half-plane $k$-arm event. All these events decay as powers of $R$ and the exact power is called the corresponding critical exponent.

Theorem 5.1. For the hexagonal lattice, we have

(i) 38] $\mathrm{P}\left(A_{R}^{1}\right)=R^{-5 / 48+o(1)}$

(ii) [53] For $k \geq 2, \mathrm{P}\left(A_{R}^{k}\right)=R^{-\left(k^{2}-1\right) / 12+o(1)}$

(iii) [53] For $k \geq 1, \mathrm{P}\left(A_{R}^{k, H}\right)=R^{-k(k+1) / 6+o(1)}$

(iv) [53] $\theta(p)=(p-1 / 2)^{5 / 36+o(1)}$

It was shown by Kesten [31] that (iv) follows from (i) and the case $k=4$ in (ii). See 43 for a detailed derivation of this.

For dynamical percolation on the hexagonal lattice, $A_{R}^{1}, A_{R}^{2}$ and $A_{R}^{1, H}$ and their exact critical exponents were relevant in the work in [51] while $A_{R}^{1}, A_{R}^{4}$, $A_{R}^{1, H}$ and even some "corner-plane" events and their exact critical exponents were relevant in the work in [14.

We finally mention that the above proofs rely on the conformal invariance of percolation on the hexagonal lattice proved by Smirnov (see [52]) and the convergence of the discrete interface in critical percolation to $S L E_{6}$ (see [9] and [52]). SLE originally stood for stochastic Löwner evolution when it was introduced by Schramm in [50] and is presently called the Schramm-Löwner evolution. It has one parameter, usually called $\kappa$, and therefore written $\mathrm{SLE}_{\kappa}$. As one varies $\kappa$, these yield random curves which describe many 2-dimensional critical systems.

\section{Exceptional times and positivity of the noise sensitivity exponent for the hexagonal lattice}

Considering the question of whether there are exceptional times for dynamical percolation in $\mathbb{Z}^{2}$, while this was not accomplished in [51, it was proved in this paper that exceptional times do exist for the hexagonal lattice. What allowed the proof to go through for the hexagonal lattice is that various exact critical exponents from critical percolation have been established for the hexagonal lattice. These same critical exponents are expected to hold for $\mathbb{Z}^{2}$ but have not at this point been established.

Theorem 6.1. 51] For critical dynamical percolation on the hexagonal lattice, there exist exceptional times of percolation and the Hausdorff dimension of the set of such times is in $[1 / 6,31 / 36]$.

As far as the noise sensitivity exponent for left to right crossing of an $n \times n$ square, the following was shown.

Theorem 6.2. 51] Consider the sequence $\left\{f_{n}\right\}$ of indicator functions for a left to right crossing of an $n \times n$ square in the hexagonal lattice. Then $\operatorname{SENS}\left(\left\{f_{n}\right\}\right) \geq 1 / 8$. For the square lattice, $\operatorname{SENS}\left(\left\{f_{n}\right\}\right)>0$. 
This was the first result where one obtained a positive lower bound on $\operatorname{SENS}\left(\left\{f_{n}\right\}\right)$ for crossing probabilities. One of the key steps in [51] is the following result which gives conditions under which one can obtain some bounds on the Fourier coefficients. We will not give any indication of its proof here. We hope that this result will be applicable in other contexts in order to bound the "level- $k$ " Fourier coefficients.

Theorem 6.3. 51

Let $f:\{0,1\}^{n} \rightarrow \mathbb{R}$. Let $A$ be a randomized algorithm determining the value of $f$. This means that $A$ examines the input bits of $f$ one by one, where the choice of the next bit examined may depend on the values of the bits examined so far and on some additional exterior randomness. The algorithm A may of course stop once it knows the output. Let $J \subseteq\{1,2, \ldots, n\}$ be the (random) set of bits examined by the algorithm. (Crucially, $J$ need not be all the bits since based on the bits examined at some point, the output might at that point be determined.) Set

$$
\delta_{A}:=\sup \{\mathrm{P}(i \in J): i \in\{1,2, \ldots, n\}\} .
$$

Then, for every $k=1,2, \ldots$, the Fourier coefficients of $f$ satisfy

$$
\sum_{|S|=k} \hat{f}(S)^{2} \leq \delta_{A} k\|f\|^{2},
$$

where $\|f\|$ denotes the $L^{2}$ norm of $f$.

We first give an outline of the proof of Theorem 6.2.

Outline of proof:

Step 1: Find a randomized algorithm to detect whether there is a left to right crossing of whites such that a fixed hexagon is looked at with probability at most $(1 / n)^{1 / 4+o(1)}$. If we place white hexagons on the right and top sides of the box and black hexagons on the bottom and left sides of the box, we can start at the bottom right and follow the path which always keeps white on the right side and blacks on the left. This is called an interface. This interface will end up either hitting the left side before the top, which implies there is a left to right white crossing or it will end up either hitting the top side before the left, which implies there is no left to right white crossing. If we "follow" this interface, revealing hexagon colors as we need to know how the interface develops, this will yield a randomized algorithm which will determine if there is a crossing. In addition, hexagons near the center will be looked at with probability at most $O(1)(1 / n)^{1 / 4+o(1)}$ since to be looked at, one must see the "2-arm" event emanating from that hexagon and one can apply Theorem 5.1(ii). This does not work however for hexagons near the boundary. To get an algorithm which looks at every hexagon with the above probability, one does some random modification of the above where one runs two interfaces from a random point on the right side. The argument then requires using the 1-arm half plane exponent as well.

Step 2: Step 1 and Theorem 6.3 gives us a bound on the sum of the "level- $k$ " 
Fourier coefficients. We plug that into (4.3) and compute. The $\mathbb{Z}^{2}$ case is similar but there we do not have the explicit critical exponents at our disposal.

Remarks:

We might hope that one can bring the value $1 / 8$ up to the suggested value of $3 / 4$ by finding better algorithms to which we can apply Theorem 6.3. However, this is not possible. As mentioned in [51, a general inequality of O'Donnell and Servedio or Theorem 6.3 applied in the case $k=1$ allows us to conclude that any algorithm will have a $\delta$ of at least $(1 / n)^{1 / 2+o(1)}$. The existence of such an algorithm would (as above) bring the value of $1 / 8$ up to $1 / 4$ and hence the best this method could yield is a noise sensitivity exponent of $1 / 4$ (unless one improves Theorem 6.3 itself). It is worth pointing out here that an algorithm which is conjecturally better than the one given in the proof of Theorem 6.2 is the one where the hexagon chosen at a given time is the one with the largest influence at the time. This is related to playing random-turn hex; see [48].

We now give an outline of the proof of Theorem 6.1.

Outline of proof:

We first explain the existence of exceptional times. We let $X_{R}:=\int_{0}^{1} 1_{V_{t, R}} d t$ where $V_{t, R}$ is the event that at time $t$ there is an open path from the origin to distance $R$ away. The key step is to show that $\mathrm{E}\left[X_{R}^{2}\right] \leq O(1) \mathrm{E}\left[X_{R}\right]^{2}$ (i.e., we use the second moment method) since from here the result is standard. Note the first moment is just $\mathrm{P}\left(A_{R}^{1}\right)$ from Section 5 . The key to getting a good bound on the second moment is to get a good bound on $\mathrm{P}\left(V_{t, R} \cap V_{0, R}\right)$. Using independence, we see this is at most $\mathrm{P}\left(V_{0, r}\right) \mathrm{P}\left(V_{t, r, R} \cap V_{0, r, R}\right)$ where $V_{t, r, R}$ is the event that at time $t$ there is an open path from distance $r$ away to distance $R$ away. We note that looking at our process at times 0 and $t$ is exactly looking at a configuration and its noisy version, which we studied earlier, with $\epsilon$ being $1-e^{-t}$. For the second factor, we use (4.3), construct a randomized algorithm for this event with a good $\delta$ (which is somewhat harder than in Theorem 6.2) and apply Theorem 6.3 to bound the relevant Fourier coefficients. The rest is algebra.

For the Hausdorff dimension, the lower bound is obtained using the calculation in the first paragraph together with Frostman's Theorem. The upper bound is easier; we use the method of proof of Theorem 2.5 together with Theorem 5.1(iv).

In [51, other types of events are also looked at such as $k$-arm events in wedges and cones and upper and lower bounds on the Hausdorf dimension of sets of exceptional times are obtained. These upper and lower bounds are however never matching. 


\section{The exact Hausdorff dimension of exceptional times and the exact noise sensitivity exponent for the hexagonal lattice}

In [14, two of the main results (among many others) were computing the exact noise sensitivity exponent for the crossing of an $n \times n$ box in the hexagonal lattice and the exact Hausdorff dimension of the set of exceptional times for dynamical percolation on the hexagonal lattice. The latter number is the upper bound obtained in Theorem 6.1.

Theorem 7.1. 14] For critical dynamical percolation on the hexagonal lattice, the Hausdorff dimension of the set of exceptional times is $31 / 36$. In addition, for critical dynamical percolation on the square lattice, there exist exceptional times of percolation.

Theorem 7.2. 14 Consider the sequence $\left\{f_{n}\right\}$ of indicator functions for a left to right crossing of an $n \times n$ square in the hexagonal lattice. Then

$$
\operatorname{STAB}\left(\left\{f_{n}\right\}\right)=\operatorname{SENS}\left(\left\{f_{n}\right\}\right)=3 / 4 .
$$

(While we will not spell these out in detail, for the square lattice, analogous results are obtained which relate $\operatorname{STAB}\left(\left\{f_{n}\right\}\right)$ and $\operatorname{SENS}\left(\left\{f_{n}\right\}\right)$ with the expected number of pivotal edges in large boxes.)

The value 31/36 was the conjectured value in [51] and the suggested value of $3 / 4$ was explained earlier. The improvements here over Theorems 6.1 and 6.2 are due almost exclusively to providing much sharper results concerning the Fourier spectrum, both for crossing probabilities and an annulus type event which is the relevant event for studying exceptional times. These arguments do not for example use Theorem 6.3 or any variant of this. This analysis is very long and intricate and so I will only say a few words about it and even for that I will stick to Theorem 7.2 .

(Very vague) Outline of proof:

The much easier direction is to show that $\operatorname{STAB}\left(\left\{f_{n}\right\}\right) \leq 3 / 4$. For a hexagon to be pivotal, there have to be, starting from that hexagon, "open paths" to the left and right sides of the box and "closed paths" to the top and bottom sides of the box. It is known that this has the same exponent as the 4-arm event. Looking at points far from the boundary and using Theorem 5.1 (ii), we obtain that $I\left(f_{n}\right) \geq n^{3 / 4+o(1)}$. In [14, it is shown that the boundary contributions can be controlled so that we indeed have $I\left(f_{n}\right)=n^{3 / 4+o(1)}$. Now Theorem 4.6 finishes the argument.

The proof that $\operatorname{SENS}\left(\left\{f_{n}\right\}\right) \geq 3 / 4$ is significantly more difficult. By Theorem 4.8, we need to prove (4.6) and so we need to obtain upper bounds on the lower tail of the distribution of $\left|\mathcal{S}_{n}\right|$. Of course, we only care about the distribution of $\left|\mathcal{S}_{n}\right|$ rather than the distribution of $\mathcal{S}_{n}$. However, it turns out that in order to study and analyze the former, it is crucial to study the latter, which has much more structure and therefore more amenable to analysis. A first key step is for general Boolean functions and gives upper bounds on

$$
\mathrm{P}(\mathcal{S} \cap B \neq \emptyset=\mathcal{S} \cap W),
$$


where $B$ and $W$ are disjoint subsets of the domain variables $1, \ldots, n_{m}$, in terms of a more general notion of pivotality. While one needs such a result for all $W$, looking at the two special cases where $W$ is $\emptyset$ or $B^{c}$ illustrates well this relationship. This general result gives in the context of percolation that if $B$ is a connected set of hexagons, $\mathrm{P}(\mathcal{S} \cap B \neq \emptyset)$ is at most 4 times the probability of having 4 alternating arms from the set $B$ out to the boundary of our $n \times n$ box and $\mathrm{P}(\emptyset \neq \mathcal{S} \subseteq B)$ is at most 4 times the previous probability squared. This starts to get the ball rolling as it relates the difficult spectral measure to things that are a little bit more concrete.

Now let $g_{r}:=r^{2} \alpha_{4}(r)$ which is close to the expected number of pivotals for a left to right crossing in an $r \times r$ box or equivalently the expected size of the spectral sample for this event. This grows to $\infty$ with $r$. One shows that

$$
\mathrm{P}\left(\left|\mathcal{S}_{n}\right|<g_{r}, \mathcal{S}_{n} \neq \emptyset\right) \asymp(n / r)^{2}\left(\alpha_{4}(n) / \alpha_{4}(r)\right)^{2} .
$$

It is not hard to show, using the fact that the 4 arm exponent is $5 / 4$, that (4.6) holds and that one can take $\delta$ to be $\gamma^{3 / 2+\epsilon}$ for any fixed $\epsilon>0$. While we want the upper bound, it is instructive to see how the lower bound is obtained which is as follows. We break the $n \times n$ square into about $(n / r)^{2} r \times r$ squares. It turns out that in the percolation context and with $B$ being an $r \times r$ square and $W=B^{c}$, the upper bound on (7.1) is shown to also be a lower bound (up to constants) and so for each $r \times r$ square $B$, the probability that the spectrum is nonempty and sits inside $B$ can been shown to be at least $\Omega(1)\left(\alpha_{4}(n) / \alpha_{4}(r)\right)^{2}$. Next it is shown that, conditioned on the spectrum intersecting such a box and no others, there is a uniform lower bound on the probability that the spectral size within that box is at most $O(1) g_{r}$. Since, as we vary the $r \times r$ square, we obtain $(n / r)^{2}$ disjoint events, we obtain the (much easier) lower bound of (7.2).

For the upper bound (which is much harder), we again break the $n \times n$ square as above and look at the number of $r \times r$ squares which intersect the spectral sample. Call this number $X_{n, r}$. Using a very difficult geometric induction argument, it is shown that

$$
\mathrm{P}\left(X_{n, r}=k\right) \leq g(k)(n / r)^{2}\left(\alpha_{4}(n) / \alpha_{4}(r)\right)^{2}
$$

where $g(k)$ grows slower than exponentially (but faster than any polynomial). It turns out that there is a "converse" of what we wrote in the previous paragraph which is that conditioned on the spectrum touching a box, there is uniform lower bound on the probability that the size of the spectrum is at least $\Omega(1) g_{r}$. This involves quite difficult percolation arguments. Given $X_{n, r}=k$, if it were the case that the sizes of the spectrum in the $k$ different $r \times r$ boxes which the spectrum hits were independent, then the probability that all of them have size less than $\Omega(1) g_{r}$ would be at most $c^{k}$ for some $c<1$. Since $\sum_{k} g(k) c^{k}<\infty$, we would be done. The problem is, under this conditioning, the spectrum in the different boxes are not independent and have a very complicated dependency structure. It is however shown that, while it is difficult to deal with the spectrum in one box conditioned on its behavior elsewhere, it is possible to deal with the spectrum in one box conditioned on it hitting that box and not intersecting some other fixed 
set. This together with a novel type of large deviations argument allows us to carry out the upper bound in (7.2).

In proving the above, other interesting results are obtained. For example, it is shown for percolation on $\mathbb{Z}^{2}$ that rerandomizing a small portion of only the vertical edges is sufficient for the correlation to go to 0 . This result suggests new interesting directions concerning noise sensitivity for Boolean functions when only some of the bits are rerandomized.

\section{Sensitivity of the infinite cluster in critical percolation}

Except in the second part of Theorem 2.4, all results in this paper so far dealt with graphs which do not percolate at criticality. It turns out that if we deal with graphs which do percolate at criticality and ask if there are exceptional times of nonpercolation, the structure of the problem is quite different. In addition, it seems to me that the set of exceptional times in this case might have similar structure to the set of "fast points" for Brownian motion; see the discussion at the end of Section 3

In [47, among other things, a fairly detailed study of this question was made for spherically symmetric trees. Special cases of the two main results of that paper are the following. In this section (only), we are dropping the assumption of homogeneous edge probabilities but will assume all edge probabilities are bounded away from 0 and 1 . The definition of $w_{n}$ from Section 3 should be modified in the obvious way.

Theorem 8.1. Consider a spherically symmetric tree with spherically symmetric edge probabilities (meaning all edges at a given level have the same retention probability). Let $w_{n}$ be as in Theorem 3.1.

(i) If

$$
\lim _{n} \frac{w_{n}}{n(\log n)^{\alpha}}=\infty
$$

for some $\alpha>2$, then there are no exceptional times of nonpercolation.

(ii) If

$$
w_{n} \asymp n(\log n)^{\alpha}
$$

for some $1<\alpha \leq 2$, then there are exceptional times of nonpercolation.

Note that in both of these regimes, Theorem 3.1 tells us that there is percolation at a fixed time.

Remarks:

(1) To see a concrete example, if we have a tree with $\left|T_{n}\right| \asymp 2^{n} n(\log n)^{\alpha}$ and $p=1 / 2$ for all edges, then if $\alpha>2$, we are in case (i) while if $\alpha \leq 2$, we are in case (ii). (Note Lyons' theorem tells us that $p_{c}=1 / 2$ in these cases.)

(2) The theorem implies that if $w_{n} \asymp n^{\alpha}$ with $\alpha>1$, then there are no exceptional times of nonpercolation, while note that if $w_{n} \asymp n$, then Theorem 3.1 implies that 
there is no percolation at a fixed time. Hence, if we only look at the case where $w_{n} \asymp n^{\alpha}$ for some $\alpha \geq 1$, we do not see the dichotomy (within the regime where we percolate at criticality) that we are after but rather we see the much more abrupt transition from not percolating at a fixed time to percolating at all times. Rather, Theorem 8.1 tells us that we need to look at a "finer logarithmic scale" to see this "phase transition" of changing from percolating at a fixed time but having exceptional times (of nonpercolation) to percolating at all times.

Interestingly, it turns out that even within the regime where there are no exceptional times of nonpercolation, there are still two very distinct dynamical behaviors of the process, yielding another phase transition.

Theorem 8.2. Consider a spherically symmetric tree $T$ of bounded degree and let $w_{n}$ be as in the previous result.

(i) When $\sum_{n=1}^{\infty} n w_{n}^{-1}<\infty$, a.s. the set of times $t \in[0,1]$ at which the root percolates has finitely many connected components. (This holds for example if $w_{n} \asymp$ $n^{\theta}$ with $\theta>2$ as well as for supercritical percolation on a homogeneous tree.)

(ii) If $w_{n} \asymp n^{\theta}$, where $1<\theta<2$, then with positive probability the set of times $t \in[0,1]$ at which the root percolates has infinitely many connected components. The same occurs if $w_{n} \asymp n(\log n)^{\alpha}$ for any $\alpha>1$.

Remarks:

(1) If $w_{n} \asymp n^{2}$, we do not know the answer. A first moment calculation suggests that there should be infinitely many connected components with positive probability but the needed inequality for a successful second moment argument fails.

(2) It is easy to show that for any graph, if there are exceptional times of nonpercolation, then the set of times $t \in[0,1]$ at which a fixed vertex percolates is totally disconnected and hence has infinitely many connected components with positive probability.

(3) We will not indicate here any proofs but we will mention one word about Theorem 8.2 since the critical exponent of 2 there derives from a difference in the ordinary model in these two regimes. Namely, the expected number of pivotal edges for the event that the root percolates is infinite for $\theta \leq 2$ but finite for $\theta>2$.

\section{Dynamical percolation and the incipient infinite cluster}

\subsection{The incipient infinite cluster}

We know that on $\mathbb{Z}^{2}$ and on the hexagonal lattice, there is no infinite cluster at $p_{c}$. Nonetheless, physicists and others have tried to talk about the "infinite cluster on $\mathbb{Z}^{2}$ containing the origin at criticality". This was made sense of by Kesten in [29] where the following result was obtained. $\Lambda_{n}$ is the box of size $n$ centered at the origin. 
Theorem 9.1. 29] The limiting measures

$$
\lim _{p \downarrow 1 / 2} \mathrm{P}_{p}(\cdot \mid 0 \leftrightarrow \infty)
$$

and

$$
\lim _{n \rightarrow \infty} \mathrm{P}_{1 / 2}\left(\cdot \mid 0 \leftrightarrow \partial \Lambda_{n}\right)
$$

both exist and are equal.

This limiting measure is referred to as the incipient infinite cluster. Properties of this were also obtained and furthermore, in [30, Kesten showed that random walk on the incipient infinite cluster is subdiffusive.

\subsection{The incipient infinite cluster and dynamical percolation}

It was asked quite early on whether the configuration for dynamical percolation at a (properly chosen) exceptional time at which the origin percolates (assuming there are such exceptional times) should have the same distribution as the incipient infinite cluster of the previous subsection. See the discussion concerning this question in [17. This question was answered in a very satisfactory manner by Hammond, Pete and Schramm in [20, a paper which is in the process of being written up. We sketch here a part of what was done in this paper.

The first key step in being able to "find" the incipient infinite cluster inside of dynamical percolation is to be able to define a local time for when the origin is percolating. There are two different approaches used to define a local time.

The first approach is as follows. Let $A_{R, t}$ be the event that at time $t$ there is an open path from the origin to distance $R$ away and let $\mathcal{T}_{R}$ be the random set of times at which $A_{R, t}$ occurs. Define a random measure $\mu_{R}$ on $\mathbb{R}$ by

$$
\mu_{R}:=\frac{1}{\alpha_{1}(R)} \mathcal{L}_{\mathcal{T}_{R}}
$$

where $\mathcal{L}_{F}$ refers to Lebesgue measure restricted to the set $F$ and $\alpha_{1}(R)=\mathrm{P}\left(A_{R, t}\right)$. Clearly, $\mathrm{E}\left[\mu_{R}([a, b])\right]=b-a$. We then let $R$ tend to infinity.

The second approach (which turns out to be equivalent) is as follows and is closer in spirit to Kesten's original definition of the incipient infinite cluster. Consider ordinary percolation and let $S$ be a collection of hexagons and let $\omega^{S}$ be the percolation realization restricted to $S$. We want to measure in some sense how much $\omega^{S}$ "helps percolation to infinity". This is made precise by the limit

$$
\lim _{R \rightarrow \infty} \frac{\mathrm{P}\left(A_{R} \mid \omega^{S}\right)}{\mathrm{P}\left(A_{R}\right)}
$$

which is easily shown to exist using Theorem 9.1

Calling this limit $f\left(\omega^{S}\right)$, let $M_{r}(\omega):=f\left(\omega^{B_{r}}\right)$ where $B_{r}$ is the ball of radius $r$ around the origin. Finally let

$$
\nu_{r}([a, b]):=\int_{a}^{b} M_{r}\left(\omega_{s}\right) d s .
$$


Theorem 9.2. 20

(i) For all $a<b, \mu_{R}([a, b])$ converges a.s. and in $L^{2}$ to a limit as $R$ goes to $\infty$, which we call $\mu_{\infty}([a, b])$.

(ii) For all $a<b, \nu_{r}([a, b])$ converges a.s. and in $L^{2}$ to a limit as $r$ goes to $\infty$, which we call $\nu_{\infty}([a, b])$.

(iii) The two above limits are a.s. the same.

Clearly the limiting measure $\mu_{\infty}$ is supported on the set of exceptional times at which the origin percolates (the latter known to be a nonempty closed set). It is not known if the support of the measure is exactly the set of exceptional times. It is explained that $\mu_{R}([a, b])$ is a martingale which implies its a.s. convergence. Using estimates from [51, one can see it is also $L^{2}$ bounded which gives the $L^{2}$ convergence. The convergence in $L^{2}$ guarantees that the limiting measure is nonzero. It is also shown that $\nu_{r}([a, b])$ is a martingale.

The final result links up the incipient infinite cluster with dynamical percolation.

Theorem 9.3. 20] Consider the random measure $\mu_{\infty}$ on $\mathbb{R}$ above and let $X$ be a Poisson process on $\mathbb{R}$ with "intensity measure" $\mu_{\infty}$. Then the distribution of $\omega_{0}$ given $0 \in X$ has the same distribution as the incipient infinite cluster.

It is not so hard to make sense of the conditioning $0 \in X$ even if this event has probability 0 ; see Chapter 11 of [27. There are a number of other results in this paper which we do not detail here.

\section{The scaling limit of planar dynamical percolation}

Before discussing the scaling limit of dynamical percolation, it is necessary to first discuss the scaling limit of ordinary percolation. There is a lot to be said here and this section will be somewhat less precise than the earlier sections. Since even the formulations can be quite technical, I will be, unlike in the previous sections, "cheating" in various places.

Even before we state the scaling limit of percolation, we need to first briefly explain the concept of conformal invariance and Cardy's formula. Let $\Omega$ be a simply connected open domain in the plane and let $A, B, C$ and $D$ be 4 points on the boundary of $\Omega$ in clockwise order. Scale a 2-dimensional lattice, such as $\mathbb{Z}^{2}$ or the hexagonal lattice, by $1 / n$ and perform critical percolation on this scaled lattice. Let $\mathrm{P}(\Omega, A, B, C, D, n)$ denote the probability that, in the $1 / n$ scaled hexagonal lattice, there is a white path of hexagons inside $\Omega$ going from the boundary of $\Omega$ between $A$ and $B$ to the boundary of $\Omega$ between $C$ and $D$. The first half of the following conjecture was stated in [36] and attributed to Michael Aizenman. The second half of the conjecture is due to Cardy [11].

Conjecture 10.1. (i) For all $\Omega$ and $A, B, C$ and $D$ as above,

$$
\mathrm{P}(\Omega, A, B, C, D, \infty):=\lim _{n \rightarrow \infty} \mathrm{P}(\Omega, A, B, C, D, n)
$$


exists and is conformally invariant in the sense that if $f$ is a conformal mapping, then $\mathrm{P}(\Omega, A, B, C, D, \infty)=\mathrm{P}(f(\Omega), f(A), f(B), f(C), f(D), \infty)$.

(ii) There is an explicit formula (not stated here) for $\mathrm{P}(\Omega, A, B, C, D, \infty)$, called Cardy's formula, when $\Omega$ is a rectangle and $A, B, C$ and $D$ are the 4 corner points. (Since every $\Omega, A, B, C$ and $D$ can be mapped to a unique such rectangle (with $A, B, C$ and $D$ going to the 4 corner points), this would specify the above limit in general assuming conformal invariance.)

Cardy's formula is quite complicated involving hypergeometric functions but Lennart Carleson realized that assuming conformal invariance, there is a nicer set of "representing" domains with four specified points for which the limit has a much simpler form. Namely, if $\Omega$ is an equilateral triangle (with side lengths 1 ), $A, B$ and $C$ the three corner points and $D$ (on the line between $C$ and $A$ ) having distance $x$ from $C$, then the above probability would just be $x$. Using Carleson's reformulation of Cardy's formula, Smirnov proved the above conjecture for the hexagonal lattice.

Theorem 10.2. 52] For the hexagonal lattice, both (i) and (ii) of the above conjecture are true.

This conjecture is also believed to hold on $\mathbb{Z}^{2}$ but is not (yet) proved in that case. In [50], Schramm described what the interfaces between whites and blacks should be as the lattice spacing goes to 0 , assuming conformal invariance. In the appropriate formulation, it should be an $\mathrm{SLE}_{6}$ curve. Smirnov [52] proved this convergence for one interface and Camia and Newman [9] proved a "full scaling limit", which is a description of the behavior of all the interfaces together. The critical exponents described in Section 5 are proved by exploiting the SLE description of the interfaces. All of the above is described well in [54.

It turns out, in order to obtain a scaling limit of dynamical percolation, a different description, due to Schramm and Smirnov, of the scaling limit of ordinary percolation is preferable. A quad $Q$ is a subset of the plane homeomorphic to a disk together with its boundary partitioned into 4 continuous pieces. A configuration for the scaling limit is described by a family of 0-1 random variables $X_{Q}$ indexed by the quads $Q$ where $X_{Q}=1$ means there is a crossing from the first to the third boundary piece of $Q$ using white hexagons. Equivalently, an element of the state space is a collection of quads (satisfying a certain necessary monotonicity condition and suitably measurable) where the collection of quads represents the quads which are "crossed". An important advantage of this state space, which we denote by $\mathcal{S}$, is that it is a compact metrizable space. This avoids the need for tightness arguments. If we perform percolation on the $1 / n$ scaled hexagonal lattice, the $X_{Q}$ 's are well-defined random variables and so we obtain a probability measure $\mu_{n}$ on $\mathcal{S}$. It turns out that the sequence of probability measures $\mu_{n}$ have a limit $\mu_{\infty}$, which we call the scaling limit. Note that for each $n$, there is essentially a 1-1 correspondence between percolation realizations on the $1 / n$ scaled lattice and elements in $\mathcal{S}$. In the limit however, we can not talk anymore about percolation 
configurations but rather the only information left is which quads are "crossed". (The latter is sort of the "macroscopic" information.)

We now move to a scaling limit for dynamical percolation. Consider dynamical percolation on the $1 / n$ scaled hexagonal lattice. We can think of our dynamical percolation on this scaled lattice as a process $\left\{\eta^{n}(t)\right\}_{t \in \mathbb{R}}$ taking values in $\mathcal{S}$. We can try to let $n$ go to infinity, in which case, the marginal for each $t$ will certainly go to $\mu_{\infty}$. However due to the noise sensitivity of percolation, for any fixed $s<t$ and any quad $Q$, the crossing of $Q$ at time $s$ and at time $t$ will become asymptotically independent as $n \rightarrow \infty$ which implies that the processes $\left\{\eta^{n}(t)\right\}_{t \in \mathbb{R}}$ converge to something which is "independent copies of $\mu_{\infty}$ for all different times". This is clearly not what we want. In order to obtain a nontrivial limit, we should slow down time by a factor of $1 /\left(n^{2} \alpha_{4}(n)\right)$ where $\alpha_{4}(n)$ is explained in Section 7

Theorem 10.3. [15] Let $\sigma_{t}^{n}:=\eta_{t n^{-2} \alpha_{4}^{-1}(n)}^{n}$. Then $\left\{\sigma^{n}(t)\right\}_{t \in \mathbb{R}}$ converges in law to a process $\left\{\sigma^{\infty}(t)\right\}_{t \in \mathbb{R}}$ under the topology of local uniform convergence. Moreover, the limiting process is Markovian (which is not apriori obvious at all).

We explain now why the time scaling is as above. Consider the quad corresponding to the unit square together with its four sides. The expected number of pivotals for a left to right crossing of this quad on the $1 / n$ scaled lattice is $\asymp n^{2} \alpha_{4}(n)$. Next, the above time scaling updates each hexagon in unit time with probability about $1 / n^{2} \alpha_{4}(n)$ and therefore, by the above, updates on average order 1 pivotals. It follows from the main result in [14] that if we scale "faster" than this, you get a limit which is independent at different times and if we scale "slower" than this, you get a limit which is constant in time. This scaling is such that the correlation between this event occuring at time 0 and occuring at time 1 stays strictly between 0 and 1 , which is something we of course want.

In the above paper, there is another model which is studied called near-critical percolation for which a scaling limit is proved in a similar fashion. Near-critical percolation can be thought of as a version of dynamical percolation, where sites are flipping only in one direction and hence after time $t$, the density of open sites is about $1 / 2+t /\left(n^{2} \alpha_{4}(n)\right)$.

To prove Theorem 10.3 there are two key steps. The first key step is a stability type result. In very vague terms, it says that if we look at the configuration at time 0 , ignore the evolution of the hexagons which are initially pivotal only for crossings which occur at macroscopic scale at most $\rho$ (which should be thought of as much larger than the lattice spacing) but observe the evolution of the hexagons which are initially pivotal for crossings which occur at macroscopic scale larger than $\rho$, then we can still predict quite well (i.e., with high probability if $\rho$ is small) how the crossings evolve on macroscopic scale 1.

Since we cannot see the individual hexagons which are initially pivotal for crossings at scale at least $\rho$ in the scaling limit, in order for this stability result to be useful, we need at least that the number of such hexagons in a given region can be read off from the scaling limit, so that we know the rate with which macroscopic crossings are changing. This is obtained by the second key step. For every $n$, look 
at ordinary percolation on the $1 / n$ scaled lattice and consider the set of hexagons from which emanate 4 paths of alterating colors to macroscopic-distance $\rho$ away. (These are the hexagons which, if flipped, change the state of a crossing at scale $\rho$.) Let $\nu_{n}^{\rho}$ be counting measure on such points divided by $n^{2} \alpha_{4}(n)$. This scaling is such that the expected measure of the unit square is of order 1 (as $n \rightarrow \infty$ if $\rho$ is fixed).

Theorem 10.4. [15] For all $\rho,\left(\omega_{n}, \nu_{n}^{\rho}\right)$ converges, as $n \rightarrow \infty$, to a limit $\left(\omega_{\infty}, \nu_{\infty}^{\rho}\right)$ and $\nu_{\infty}^{\rho}$ is a measurable function of $\omega_{\infty}$.

The argument of this result is very difficult. I say however a word on how this gives a nice construction of the scaling limit of dynamical percolation. The idea is that one constructs a dynamical percolation realization by first taking a realization from the $t=0$ scaling limit (or equivalently $\omega_{\infty}$ above), looks at $\nu_{\infty}^{\rho}$ (which requires no further randomness because $\nu_{\infty}^{\rho}$ has been proved to be a function of $\omega_{\infty}$ ) and then builds an appropriate Poisson point process over the random measure $\nu_{\infty}^{\rho} \times d t$ which will be used to dictate when the different crossings of quads of scale at least $\rho$ will change their state. This is done for all $\rho$ and the stability result is needed to ensure that the process described in this way is in fact the scaling limit of dynamical percolation. The idea that dynamical percolation (and near-critical percolation) could possibly be built up in this manner from a Poisson process over a random measure was suggested by Camia, Fontes and Newman in the paper [10.

It is also shown in [15] that a type of "conformal covariance" for the above measures $\nu_{\infty}^{\rho}$ holds but this will not be detailed here. The argument for Theorem 10.4 also proves the existence of natural time-parametrizations for the $\mathrm{SLE}_{6}$ and $\mathrm{SLE}_{8 / 3}$ curves, a question studied in [37] for general $\mathrm{SLE}_{\kappa}$.

\section{Dynamical percolation for interacting particle systems}

In [7, certain interacting particle systems were studied and the question of whether there are exceptional times at which the percolation structure looks different from that at a fixed time was investigated. The two systems studied were the contact process and the Glauber dynamics for the Ising model. Most of the results dealt with noncritical cases but since the dynamics are not independent, a good deal more work was needed compared to the easy proof of Proposition 2.1.

However, there was one very interesting case left open in this work which was the following. Consider the Glauber dynamics for the critical 2-dimensional Ising model. First note that 2 dimensions is special for the Ising model compared to higher dimensions since it is known that for $\mathbb{Z}^{2}$ the critical value for phase transition is the same as the critical value for when percolation occurs. The Ising model does not percolate in 2 dimensions at the critical value and in view of Theorem [6.1, it is natural to ask if there are exceptional times of percolation for the Glauber dynamics. During a visit to Microsoft, Gábor Pete suggested to me that this might not be the case since the scaling limit of the Ising model 
was conjectured to be $\mathrm{SLE}_{3}$, which unlike $\mathrm{SLE}_{6}$ (the scaling limit of percolation), does not hit itself. This yields that there should not be so many pivotal sites which suggests no exceptional times. A few months later in Park City, Christophe Garban sketched out a "1st moment argument" for me which would give no exceptional times under certain assumptions.

In any case, whether or not one can actually prove this, it now seems clear that there are no exceptional times for the Glauber dynamics. This seems to be a beautiful example of how the qualitative difference in the behavior of the two respective SLE scaling limits (self-intersections versus not) yields a fundamental difference between the natural dynamics on ordinary percolation and the natural

dynamics on the Ising model concerning whether there are exceptional times of percolation or not.

\section{References}

[1] Adelman, O., Burdzy, K. and Pemantle, R. Sets avoided by Brownian motion. Ann. Probab. 26, (1998), 429-464.

[2] Aizenman, M., Kesten, H. and Newman, C. M. Uniqueness of the infinite cluster and continuity of connectivity functions for short- and long-range percolation. Comm. Math. Phys. 111, (1987), 505-532.

[3] Benjamini, I., Häggström, O., Peres, Y. and Steif, J. Which properties of a random sequence are dynamically sensitive? Ann. Probab. 31, (2003), 1-34.

[4] Benjamini, I., Kalai, G. and Schramm, O. Noise sensitivity of Boolean functions and applications to percolation. Inst. Hautes Études Sci. Publ. Math. 90, (1999), 5-43.

[5] Benjamini, I. and Schramm, O. Exceptional planes of percolation. Probab. Theory Related Fields 111, (1998), 551-564.

[6] Berg, J. van den, Meester, R. and White, D. G. Dynamic Boolean models. Stochastic Process. Appl. 69, (1997), 247-257.

[7] Broman, E. I. and Steif J. E. Dynamical Stability of Percolation for Some Interacting Particle Systems and $\epsilon$-Movability. Ann. Probab. 34, (2006), 539-576.

[8] Burton, R. and Keane, M. Density and uniqueness in percolation. Comm. Math. Phys. 121, (1989), 501-505.

[9] Camia, F. and Newman, C. M. Two-dimensional critical percolation: the full scaling limit, Comm. Math. Phys. 268, (2006), 1-38.

[10] Camia, F., Fontes, L.R.G. and Newman, C. M. Two-dimensional scaling limits via marked nonsimple loops. Bull. Braz. Math. Soc. (N.S.) 37, (2006), 537-559.

[11] Cardy, J.L. Critical percolation in finite geometries. J. Phys. A 25, (1992), L201L206.

[12] Evans, S. N. Local properties of Levy processes on a totally disconnected group. J. Theoret. Probab. 2, (1989), 209-259.

[13] Garban, C. Oded Schramm's contributions to noise sensitivity (preliminary title), in preparation.

[14] Garban, C., Pete, G. and Schramm, O. The Fourier Spectrum of Critical Percolation, preprint, arXiv:0803.3750[math:PR]. 
[15] Garban, C., Pete, G. and Schramm, O. Scaling limit of near-critical and dynamical percolation, in preparation.

[16] Grimmett, G. Percolation. Second edition, Springer-Verlag, (1999), New York.

[17] Häggström, O. Dynamical percolation: early results and open problems. Microsurveys in discrete probability (Princeton, NJ, 1997), 59-74, DIMACS Ser. Discrete Math. Theoret. Comput. Sci., 41, Amer. Math. Soc., Providence, RI, 1998.

[18] Häggström, O. and Pemantle, R. On near-critical and dynamical percolation in the tree case. Statistical physics methods in discrete probability, combinatorics, and theoretical computer science (Princeton, NJ, 1997). Random Structures Algorithms 15, (1999), 311-318.

[19] Häggström, O., Peres, Y. and Steif, J. E. Dynamical percolation. Ann. Inst. Henri Poincaré, Probab. et Stat. 33, (1997), 497-528.

[20] Hammond, A., Pete, G. and Schramm, O. Local time for exceptional dynamical percolation, and the incipient infinite cluster, in preparation.

[21] Hara, T. and Slade, G. (1994) Mean field behavior and the lace expansion, in Probability Theory and Phase Transitions, (ed. G. Grimmett), Proceedings of the NATO ASI meeting in Cambridge 1993, Kluwer.

[22] Harris, T. E. A lower bound on the critical probability in a certain percolation process. Proc. Cambridge Phil. Soc. 56, (1960), 13-20.

[23] Jonasson, J. Dynamical circle covering with homogeneous Poisson updating Statist. Probab. Lett., to appear.

[24] Jonasson, J. and Steif, J. E. Dynamical models for circle covering: Brownian motion and Poisson updating. Ann. Probab. 36, (2008), 739-764.

[25] Kahn, J., Kalai, G. and Linial, N. The influence of variables on boolean functions. 29th Annual Symposium on Foundations of Computer Science, (1988), 68-80.

[26] Kalai, G. and Safra, S. Threshold phenomena and influence: perspectives from mathematics, computer science, and economics. Computational complexity and statistical physics, 25-60, St. Fe Inst. Stud. Sci. Complex., Oxford Univ. Press, New York, 2006.

[27] Kallenberg, O. Foundations of modern probability. Second edition. Probability and its Applications (New York). Springer-Verlag, New York, 2002.

[28] Kesten, H. The critical probability of bond percolation on the square lattice equals $\frac{1}{2}$. Comm. Math. Phys. 74, (1980), 41-59.

[29] Kesten, H. The incipient infinite cluster in two-dimensional percolation. Probab. Theory Related Fields 73, (1986), 369-394.

[30] Kesten, H. Subdiffusive behavior of random walk on a random cluster. Ann. Inst. Henri Poincaré, Probab. et Stat. 22, (1986), 425-487.

[31] Kesten, H. Scaling relations for 2D-percolation. Commun. Math. Phys. 109, (1987), $109-156$.

[32] Kesten, H. and Zhang, Y. Strict inequalites for some critical exponents in 2Dpercolation. J. Statist. Phys. 46, (1987), 1031-1055.

[33] Khoshnevisan D. Dynamical percolation on general trees. Probab. Theory Related Fields 140, (2008), 169-193.

[34] Khoshnevisan, D. Multiparameter processes. An introduction to random fields. Springer Monographs in Mathematics. Springer-Verlag, New York, 2002. 
[35] Khoshnevisan, D., Peres, Y. and Xiao, Y. Limsup random fractals. Electron. J. Probab. 5, (2000), no. 5, 24 pp. (electronic).

[36] Langlands, R., Pouliot, P. and Saint-Aubin, Y. Conformal invariance in twodimensional percolation. Bull. Amer. Math. Soc. (N.S.) 30, (1994), 1-61.

[37] Lawler, G. Dimension and natural parametrization for SLE curves.

[38] Lawler, G., Schramm, O. and Werner, W. One-arm exponent for critical 2D percolation. Electron. J. Probab. 7, (2002), no. 2, 13 pp. (electronic).

[39] Lyons, R. Random walks and percolation on trees. Ann. Probab. 18, (1990), 931-958.

[40] Lyons, R. Random walks, capacity, and percolation on trees. Ann. Probab. 20, (1992), 2043-2088.

[41] Lyons, R. with Peres, Y. (2008). Probability on Trees and Networks. Cambridge University Press. In preparation. Current version available at http://mypage.iu.edu/ ${ }^{\sim}$ rdlyons/

[42] Mossel, E. and O'Donnell, R. On the noise sensitivity of monotone functions. Random Structures Algorithms 23, (2003), 333-350.

[43] Nolin, P. Near-critical percolation in two dimensions. Electron. J. Probab. 13, (2008), no. $55,1562-1623$.

[44] O'Donnell, R. Computational applications of noise sensitivity, Ph.D. thesis, MIT (2003). Version available at http://www.cs.cmu.edu/ odonnell/

[45] Peres, Y. Intersection-equivalence of Brownian paths and certain branching processes. Commun. Math. Phys. 177, (1996), 417-434.

[46] Peres, Y. Remarks on intersection-equivalence and capacity-equivalence. Ann. Inst. Henri Poincaré (Physique théorique) 64, (1996), 339-347.

[47] Peres, Y., Schramm, O. and Steif, J. E. Dynamical sensitivity of the infinite cluster in critical percolation. Ann. Inst. Henri Poincaré, Probab. et Stat., to appear.

[48] Peres, Y., Schramm, O., Sheffield, S. and Wilson, D. B. Random-turn hex and other selection games. Amer. Math. Monthly 114, (2007), 373-387.

[49] Peres, Y. and Steif, J. E. The number of infinite clusters in dynamical percolation. Probab. Theory Related Fields 111, (1998), 141-165.

[50] Schramm, O. Scaling limits of loop-erased random walks and uniform spanning trees. Israel J. Math. 118, (2000), 221-288.

[51] Schramm, O. and Steif, J. E. Quantitative noise sensitivity and exceptional times for percolation. Ann. Math., to appear.

[52] Smirnov, S. Critical percolation in the plane: conformal invariance, Cardy's formula, scaling limits. C. R. Acad. Sci. Paris Sér. I Math. 333, (2001), 239-244.

[53] Smirnov, S. and Werner, W. Critical exponents for two-dimensional percolation. Math. Res. Lett. 8, (2001), 729-744.

[54] Werner, W. Lectures on two-dimensional critical percolation. IAS Park City Graduate Summer School, 2007, arXiv:0710.0856 [math:PR].

\section{Acknowledgments}

I would like to thank Erik Broman, Christophe Garban, Olle Häggström, Alan Hammond, Russell Lyons and Gábor Pete for very many helpful comments on earlier versions of the manuscript. I appreciate in particular Christophe Garban, 
Alan Hammond and Gábor Pete explaining to me their unpublished work. A part of this work was done while visiting the Mittag-Leffler Institute in Djursholm, Sweden, the same institute where dynamical percolation was started.

Jeffrey E. Steif

Mathematical Sciences

Chalmers University of Technology

and

Mathematical Sciences

Göteborg University

SE-41296 Gothenburg, Sweden

e-mail: steif@chalmers.se 